\title{
L'optique diffractive au service de la correction des aberrations
}

\author{
P. Chavel \\ Institut d'Optique Théorique et Appliquée, URA 14 du CNRS, Université de Paris Sud, \\ B.P. 147, 91403 Orsay cedex, France
}

\begin{abstract}
Résumé : après avoir rappelé l'analogie entre le réseau de diffraction, périodique suivant un axe, et le réseau zoné, a périodicité radiale, on montre que la dispersion du réseau se traduit pour le réseau zoné par un chromatisme particulièrement adapté a l'achromatisation des lentilles. On examine les méthodes de fabrication et la perturtation par les ordres parasites, puis on généralise les principes ainsi introduits a la correction d'une aberration queloonque par la fonction diffractive dans les "optiques hybrides".
\end{abstract}

Il existe deux axes de développernent rapide de l'optique diffractive.

- D'une part, la nécessité d'intégrer les systèmes opto-électroniques impose de trouver des techniques de miniaturisation de l'ensemble des fonctions nécessaires dans les systèmes optiques. Les composants diffractifs constituent l'une des approches prometteuses. C'est grâce aux technologies de la micro-électronique que peuvent apparaître des solutions nouvelles par la gravure de structures de dimensions caractéristiques très voisines de la longueur d'onde.

- D'autre part, l'incorporation d'une dose de diffraction dans des systèmes optiques permet une amélioration de performance. Il faut entendre par là un gain dans le compromis bien connu entre encombrement, poids, champ et qualité d'image. Les méthodes de fabrication relèvent ici aussi bien de l'atelier d'optique que de la microlithographie. De telles combinaisons sont dites hybrides parce que la mise en forme des fronts d'onde y procède à la fois de la réfraction (ou de la réflexion) et de la diffraction. C'est à ce second aspect qu'est consacré le présent chapitre.

\section{DU RÉSEAU AU RÉSEAU ZONÉ}

\subsection{Rappel sur les réseaux}

Nous commencerons par un rappel de quelques propriétés de bases des réseaux de diffraction, composants optiques périodiques suivant une direction. La loi bien connue des réseaux,

$$
\sin i_{q}-\sin i_{o}=\frac{q \lambda}{p}
$$


indique l'angle d'émergence de l'ordre q d'un réseau de pas p éclairé à la longueur d'onde $\lambda$ par une onde plane sous l'incidence $i_{\alpha}$. Elle résulte du théorène de Floquet [1], dont nous résumerons la teneur de la façon suivante : on considère une onde plane monochromatique dont le champ électrique s'écrit

$$
\mathbf{E}=\mathbf{E}_{\mathrm{o}} \exp \boldsymbol{i k} \cdot \mathbf{r}, \operatorname{avec} \mathbf{k}\left(k \sin i_{o}, 0 . k \cos i_{o}\right),
$$

où $\mathbf{k}$ désigne le vecteur d'onde dans le vide, où l'axe z est normal au réseau et où $\mathrm{xOz}$ est choisi comme plan d'incidence. Si cette onde rencontre une structure périodique selon l'axe $x$, elle donne lieu à un champ diffracté affecté par cette périodicité :

$$
\mathbf{E}=\sum_{q \in \boldsymbol{Z}} \mathbf{E}_{q} \exp i \mathbf{k}_{q} \cdot \mathbf{r}, \operatorname{avec}_{q}\left(k \sin i_{o}+\frac{q}{p}, 0, k \cos i_{q}\right) .
$$

L'angle $i_{q}$, éventuellement complexe si l'ordre $m$ est évanescent, est donné en imposant au module du vecteur d'onde $\mathbf{k}_{\mathrm{q}}$ d'être égal à celui de l'onde incidente.

La résolution des équations de Maxwell permet dans tous les cas de trouver l'expression des amplitudes $\mathbf{E}_{\mathrm{q}}$ des différents ordres, mais peut faire intervenir des méthodes analytiques for complexes lorsque le pas n'est pas très différent de la longueur d'onde [2]. Dans ce chapitre, nous nous limiterons au cas où l'approximation scalaire et l'approximation de la transmission sont valables, ce qui correspond à un réseau suffisamment mince et de pas nettement plus grand que la longueur d'onde. Les différentes composantes du champ $\mathbf{E}$ ont alors le même comportement, si bien que le champ électromagnétique de la lumière peut être ramené à un scalaire appelé amplitude complexe $U(x, y, z)$. Cette demière est exprimée à la sortie du réseau par le produit de l'amplitude incidente et de la "transmittance en amplitude" $t(x)$ du réseau, fonction périodique en $\mathrm{x}$ de période $\mathrm{p}$. Un formalisme semblable s'applique pour l'onde réfléchie. Considérons un réseau entièrement compris dans l'espace $0<\mathrm{z}<\mathrm{z}_{\mathrm{o}}$. La fonction $\mathrm{t}$ rend compte du déphasage et de l'absorption à la traversée du réseau. Notamment, si en un point $x$, l'épaisseur $z_{0}$ est occupée par un milieu transparent d'indice $n$ dans la région $0<z<e(x)$ et par de l'air (d'indice 1) dans la région $e(x)<z<z_{0}$, cette fonction s'écrit, en supposant la réflexion négligeable :

$$
t(x)=\exp 2 i \pi \frac{(n-1) e(x)}{\lambda}
$$

On peut alors écrire le champ à la sortie du réseau sous la forme :

$$
U\left(\mathbf{r}_{0}\right)=U_{0} \operatorname{explk} \cdot \mathbf{r}_{0} r(x) \text { avec } \mathbf{r}_{0}\left(x, 0, z_{0}\right)
$$

et en exprimant la fonction périodique t par sa série de Fourier

$$
U\left(\mathbf{r}_{o}\right)=U_{o} \sum_{q \in \Sigma} t_{q} \exp i \mathbf{k}_{q} \cdot \mathbf{r}_{o}
$$

d'où l'on déduit que l'efficacité $\eta_{q}$ de l'ordre $q$ d'un réseau dans cette approximation n'est autre que le carré du module du coefficient de Fourier correspondant de la transmittance :

$$
\eta_{q}=\left|t_{a}\right|^{2}=\left|\frac{1}{p} \int_{0}^{p} r(x) \exp 2 i \pi \frac{q x}{p} d x\right|^{2}
$$




\subsection{Les réseaux zonếs}

Considérons maintenant un composant décrit par une transmittance $t(x, y)$ périodique non plus par rapport à la variable $x$, mais par rapport au carré $\rho^{2}$ de la distance à l'axe $z(x=y=0)$ et éclairons-le par une onde plane en incidence normale : un raisonnement en tout point analogue à celui résumé par les équations (5) à (7) mène à une nouvelle décomposition :

$$
\begin{aligned}
& U(\rho, 0)=U_{0} \\
& U\left(\rho, z_{0}\right)=U_{0} \sum_{q \in 2} t_{q} \exp 2 i \pi \frac{q \rho^{2}}{\rho_{1}^{2}} \\
& \text { avec } t_{q}=\frac{1}{\rho_{1}^{2}} \int_{0}^{\rho^{2}} t(\rho) \exp -2 i \pi \frac{q \rho^{2}}{\rho_{1}^{2}} d \rho^{2}
\end{aligned}
$$

Dans ces équations, $U_{0}$ désigne l'amplitude incidente sur le composant. Sa transmittance $t$, ne dépendant que de la distance $\rho$ à l'axe, est notée comme fonction de $\rho$ pour simplifier, et $\rho_{1}$ désigne la distance à l'axe de la fin de la première période en $\rho^{2}$, encore appelée rayon de la première "zone". Le composant est ainsi constitué d'un disque entouré de zones annulaires concentriques. Les rayons des limites de zones croissent comme les racines carrées des entiers, exactement comme ceux des anneaux de Newton. Le composant porte le nom de réseau zoné, par extension du "réseau zoné de Soret" [3] que ce dernier avait réalisé par photographie d'un dessin à l'encre de Chine dans le but d'observer certaines des propriétés que l'on va établir cidessous. On parle aussi souvent de "réseau zoné de Fresnel", dénomination quelque peu abusive mais qui rappelle que les lieux des points d'un plan (ou d'une sphère) dont les distances à un point donné de l'espace differrent de moins de $\lambda / 2$ ont été introduits par ce dernier dans ses travaux fondateurs sur la diffraction [4]. La dénomination "lentille de Fresnel" est ambiguë car Fresnel ne semble pas avoir jamais utilisé de réseau zoné et qu'il a en revanche introduit à l'occasion de ses travaux sur les lentilles de phare des composants d'aspect fort semblable mais n'utilisant que l'optique géométrique et non la diffraction ; l'expression "lentille de Fresnel diffractive", historiquement inexacte, est cependant acceptable dans la mesure où elle rend hommage au pionnier du domaine. Montrons en effet que les réseaux zonés ont des propriétés très voisines de celles des lentilles.

Le développement de Fourier de l'onde sortant du réseau zoné exprimé par l'équation (8) permet d'y distinguer des ordres dans le mêrme sens qu'un réseau ordinaire possède des ordres. Toutefois, chacun de ces ordres s'exprime comme une onde sphérique : rappelons en effet que la distance entre une sphère et son plan tangent s'exprime au premier ordre non nul, appelé "approximation de Fresnel", sous la forme $\frac{\rho^{2}}{2 R}$. Dans cette expression, $\rho$ est la distance au point de contact et $\mathrm{R}$ le rayon de la sphère. Dans cette même approximation une onde sphérique

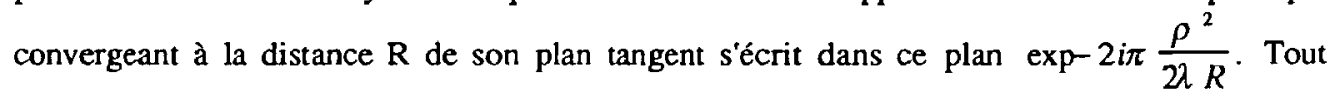
comme chaque ordre d'un réseau a l'effet d'un prisme, chaque ordre du réseau zoné se comporte ainsi comme une lentille mince de focale image

$$
f_{q}^{\prime}=\frac{\rho_{1}^{2}}{2 q \lambda}
$$

On peut montrer que cette propriété s'étend bien à une onde incidente quelconque et donc aux propriétés d'imagerie des lentilles. Dans le cas général, l'onde incidente considérée ici permet d'observer sur l'axe du réseau zoné une série de foyers aux distances indiquées par 
l'équation (9) et transportant une quantité d'énergie $\eta_{q}$ : c'est ce que présente la Figure 1 dans le cas (peu réaliste) d'un réseau qui ne présenterait que les ordres 1 et 2 , tous deux de même efficacité de diffraction. Dans la limite où aucune aberration n'intervient, la tache de diffraction formée par chaque ordre est une tache d'Airy déterminée par la pupille qui limite le réseau zoné mais qui interfere, il est vrai, avec la lumière défocalisée des autres ordres.

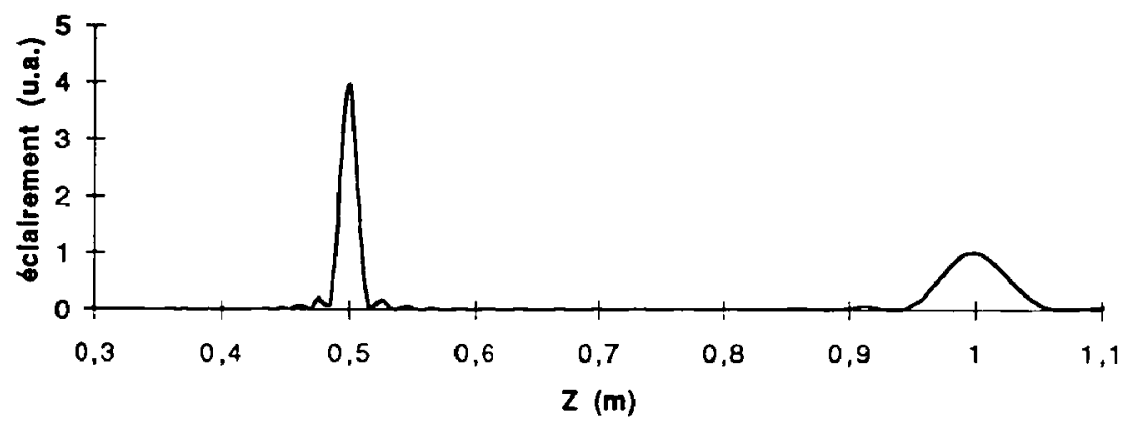

Figure 1. Diffraction sur l'axe d'un réseau zoné à deux ordres (réseau constitué de 15 zones).

\section{APPLICATION : LE DOUBLET ACHROMATIQUE HYBRIDE}

\subsection{Rappels sur l'achromatisation}

L'équation (9) met en lumière l'intérêt essentiel des réseaux zonés dans les systèmes optiques : son chromatisme tout à fait inhabituel. La focale est en effet inversement proportionnelle à la longueur d'onde, ce qui représente une dispersion à la fois beaucoup plus forte que celle des verres et de signe contraire. L'application immédiate est la réalisation de systèmes optiques achromatiques, ce qui est tout à fait important puisqu'on sait que le chromatisme est le défaut le plus gênant des lentilles simples utilisées dans le visible. Rappelons donc quelques données nécessaires et le principe de base des doublets achromatiques [5]. Nous nous limiterons au cas idéal de lentilles minces (diffractives ou réfractives) sans autre défaut que le chromatisme.

La dispersion d'indice des verres est donnée par les fabricants sous la forme de fractions rationnelles en $\lambda$, mais peut être résumée dans une première approche par deux nombres, la constringence $v$ et la dispersion secondaire $P$, définis à partir des valeurs de l'indice $n_{m}, n_{o}$ et $n_{M}$ à trois longueurs d'onde de référence notées $\lambda_{m}, \lambda_{0}$ et $\lambda_{M}$ choisies pour représenter respectivement l'extrémité inférieure, le milieu et l'extrémité supérieure de la bande de longueur d'onde jugée intéressante :

$$
v=\frac{n_{o}-1}{n_{m}-n_{M}} \text { et } P=\frac{n_{m}-n_{o}}{n_{m}-n_{M}} .
$$

A titre d'exemple, on utilise en général dans le visible comme longueurs d'onde de référence les raies spectrales conventionnellement notées $F$, $d$ et $C$. Leurs longueurs d'onde valent respectivement $\lambda_{\mathrm{F}}=486,1 \mathrm{~nm}, \lambda_{\mathrm{o}}=587,6 \mathrm{~nm}$ et $\lambda_{\mathrm{C}}=656,3 \mathrm{~nm}$. 
Pour concevoir un achromat à partir de deux lentilles minces désignés par les indices 1 et 2 , on impose :

- d'une part, la puissance pour la longueur d'onde centrale :

$$
\frac{1}{f_{0}^{\prime}}=\frac{1}{f_{01}^{\prime}}+\frac{1}{f_{02}^{\prime}} \text { que nous noterons } C_{0}=C_{01}+C_{02} \text {, }
$$

la lettre $\mathrm{C}$ représentant la convergence et $\mathrm{C}_{\mathrm{o}}$ étant donc une donnée du problème ; - d'autre part, le repliement des foyers des longueurs d'onde extrêmes :

$$
f_{m}^{\prime}=f_{M}^{\prime} \text { cest-à-dire } C_{m 1}+C_{m 2}=C_{M 1}+C_{M 2} \text {. }
$$

Dans le cas de lentilles réfractives, la focale obeit à l'expression usuelle :

$$
C=\frac{1}{f^{\prime}}=\left(n_{\lambda}-1\right)\left(\frac{1}{R^{\prime}}-\frac{1}{R^{\prime \prime}}\right)=A\left(n_{\lambda}-1\right),
$$

où la constante A est introduite pour désigner de façon abrégée les caractéristiques géométriques de la lentille, c'est-à-dire l'effet des rayons de courbures $R^{\prime}$ et $R^{\prime \prime}$ de ses deux faces. Le système des trois dernières équations fournit la solution suivante, où les indices 1 et 2 désignent toujours les deux lentilles composant le doublet et l'absence d'indice, le doublet entier :

$$
C_{o 1}=C_{o} \frac{v_{1}}{v_{1}-v_{2}} \text { et } C_{o 2}=C_{o} \frac{v_{2}}{v_{2}-v_{1}} .
$$

Le chromatisme secondaire, différence entre la convergence aux extrémités du spectre et celle à la longueur d'onde centrale, a pour valeur

$$
C_{m}-C_{o}=C_{M}-C_{o}=C_{o} \frac{P_{1}-P_{2}}{v_{1}-v_{2}}
$$

On constate que, tous les verres ayant une constringence positive, la correction est toujours obtenue en réalisant une lentille plus convergente que la consigne (en valeur absolue) dans $\mathbf{k}$ verre le plus constringent (ce qui veut dire le moins dispersif) et en compensant son chromatisme par une lentille de convergence opposée de l'autre verre : une telle situation n'est pas idéale pour la courbure des faces ni donc pour les aberrations géométriques!

\subsection{La formule du doublet achromatique hybride}

L'idée de l'achromatisation par optique diffractive s'est beaucoup développée depuis une dizaine d'années sous l'impulsion de travaux de MIT Lincoln Laboratories [6]. Il suffit de constater que la convergence d'un réseau zoné dans l'ordre q s'écrit

$$
C_{q}=\frac{2 q \lambda}{\rho_{1}^{2}}=\frac{2 q \lambda_{0}}{\rho_{1}^{2}} \frac{\lambda}{\lambda_{0}}=A\left(n_{\text {dif }}-1\right) \text { avec } n_{\text {dif }}=\frac{\lambda}{\lambda_{0}}+1
$$

pour que tout le calcul précédent s'applique. A est toujours une constante liée à la géométrie. Tout se passe comme si un composant diffractif avait un indice, $n_{d i f}$. L'intervention du $\lambda_{0}$ dans son expression est arbitraire et ne sert qu'à éviter d'outrager l'homogénéité des équations chère au physicien. La constringence et la dispersion secondaire d'un élément diffractif s'écrivent alors 


$$
v=\frac{\lambda_{0}}{\lambda_{m}-\lambda_{M}} \text { et } P=\frac{\lambda_{m}-\lambda_{o}}{\lambda_{m}-\lambda_{M}} .
$$

Le tableau 1 regroupe quelques valeurs numériques d'achromats et met en évidence les caractéristiques suivantes :

- la constringence diffractive étant toujours négative, on réalise les doublets achromatiques hybrides par addition de deux convergences de même signe, ce qui est favorable pour les aberrations géométriques :

- la diffraction est utilisable dans les domaines où les matériaux optiques sont rares, chers, difficiles à travailler ou de transparence médiocre : c'est notamment le cas de l'infrarouge thermique, d'où la présence dans le tableau de deux calculs concemant les bandes 3-5 $\mu \mathrm{m}$ et 8$12 \mu \mathrm{m}$, qui sont deux domaines de transparence d'usage courant pour l'imagerie infrarouge : - le nombre de zones des réseaux zonés peut parfois être très faible, notamment bien sûr aux faibles nombres d'ouverture, ce qui rend leur réalisation relativement aisée ;

- bien sûr, si le doublet hybride peut être réalisé par gravure d'une des faces de sa partie réfractive, il en résulte une simplification et un allégement du système optique.

L'étude qui vient d'être menée pour l'achromatisation d'un doublet présente une forte analogie avec celle de l'athermalisation d'un doublet, c'est-à-dire du calcul de la combinaison de verres qui garde la mise au point lorsqu'un système optique se dilate par échauffement. L'optique diffractive peut également présenter des avantages dans ce cas.

Tableau 1

\begin{tabular}{|c|c|c|}
\hline région spectrale & visible & infrarouge thermique \\
\hline longueur d'onde centrale $\lambda$ & $587,6 \mathrm{~nm}$ (raie d) & $10 \mu \mathrm{m}$ \\
\hline $\begin{array}{l}\text { Iongueurs d'onde extrêmes choisies pour replier } \\
\text { le chromatisme }\end{array}$ & $\begin{array}{c}486,1 \text { et } 656,3 \mathrm{~nm} \\
\text { (raies } F \text { et } C \text { ) }\end{array}$ & $8 \mathrm{et} 12 \mu \mathrm{m}$ \\
\hline focale & $100 \mathrm{~mm}$ & $200 \mathrm{~mm}$ \\
\hline nombre d'ouverture (focale/diamètre) & 4 & 1,5 \\
\hline matériau 1 , indice à $\lambda_{0}$, constringence $v$ & $\begin{array}{l}\text { verre B } 1664 ; \\
1,516 ; 64\end{array}$ & $\begin{array}{l}\text { germanium; } \\
4 ; 1000\end{array}$ \\
\hline matériau 2 , indice à $\lambda_{0}$, constringence $v$ & $\begin{array}{l}\text { verre C2036; } \\
1,620 ; 36\end{array}$ & $\begin{array}{l}\text { sulfure de zinc; } \\
\qquad 2,2 ; 100\end{array}$ \\
\hline constringence effective de la solution diffractive & $-3,45$ & $-2,5$ \\
\hline $\begin{array}{l}\text { focales des deux lentilles simples de l'achromat } \\
\text { refractif matériau 1/matériau } 2\end{array}$ & 43,$8 ;-77,8 \mathrm{~mm}$ & $180 ;-1800 \mathrm{~mm}$ \\
\hline $\begin{array}{c}\text { focales des parties réfractive (matériau 1) et } \\
\text { diffractive de l'achromat hybride }\end{array}$ & 105,$4 ; 1953 \mathrm{~mm}$ & 200,$5 ; 80000 \mathrm{~mm}$ \\
\hline nombre de zones de la partie diffractive & 68 & $\overline{3}$ \\
\hline
\end{tabular}

\subsection{Technologie et efficacité de diffraction}

Il convient de compléter notre étude par l'examen des moyens de réalisation de la gravure diffractive et de leur impact sur l'efficacité de diffraction.

Nous distinguerons deux technologies, le "masquage" et l'usinage à l'outil diamanté. Pour en comprendre le fonctionnement, il convient d'abord de s'interroger sur la possibilité d'obtenir une efficacité de $100 \%$ avec un composant diffractif. 


\subsubsection{Réseau zoné "blazé"}

De la dernière ligne de l'expression (8), il résulte que l'efficacité ne peut atteindre $100 \%$, c'està-dire que $t_{q}$ ne peut avoir un module unitaire, que si le profil $t(\rho)$ de chaque période vaut à une constante près

$$
t(\rho)=\exp 2 i \pi \frac{q \rho^{2}}{\rho_{1}^{2}}
$$

ce qui veut dire que le réseau zoné doit être de phase pure et avoir exactement le même relief qu'une lentille, limité toutefois à un déphasage de $2 \pi q$ par zone (voir Figure 2), c'est-à-dire à une hauteur

$$
h=\frac{q \lambda}{n-1}
$$

Comme il s'agit de réaliser une telle forme, ou tout au moins de s'en approcher, par gravure et non pas par les méthodes habituelles de découpe et polissage de l'atelier d'optique, l'opération est d'autant plus facile que q est faible, sauf dans les cas où les anneaux externes deviennent trop minces pour la technique utilisée. On choisit donc habituellement de travailler dans l'ordre $\mathrm{q}=1$ : comme nous le démontrons maintenant, le désir de bonnes performances sur une large bande spectrale conduit à la même conclusion.

a)

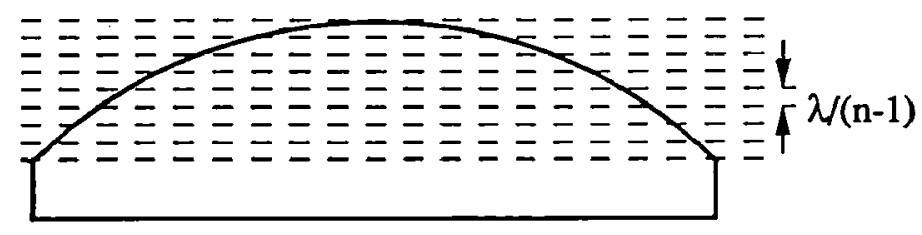

b)

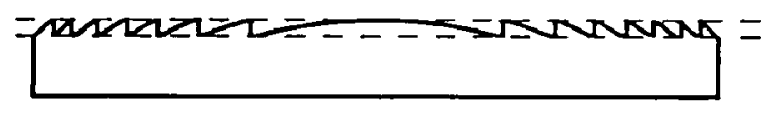

c)

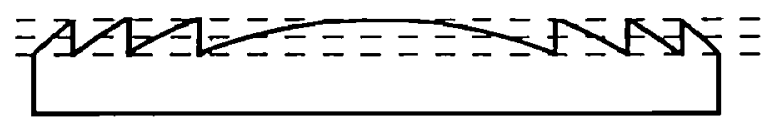

Figure 2. Profils de réseaux zonés pour optique hybride.

\subsubsection{Chromatisme de l'efficacité de diffraction}

La relation (19) ne peut être vérifiée qu'à une longueur d'onde $\lambda_{\mathrm{o}}$ donnée, que nous appellerons longueur d'onde nominale. Elle correspond en général - mais pas nécessairement - à la longueur d'onde centrale du doublet achromatique hybride considéré, c'est pourquoi nous utilisons la même notation que pour la longueur d'onde centrale des calculs d'achromatisation. L'efficacité de diffraction à toute autre longueur d'onde souffre du fait que l'interférence entre les zones n'est pas tout à fait constructive, leur déphasage n'étant pas un multiple entier de $2 \pi$. 
Le calcul à partir des équations (4) et (8) amène au profil d'épaisseur de chaque zone puis, en appelant $n$ et $n_{0}$ les indices aux longueurs d'onde $\lambda$ et $\lambda_{\mathrm{o}}$ respectivement, à l'efficacité

$$
\eta_{q}=\operatorname{sinc}^{2} q\left(1-\frac{\lambda_{0}(n-1)}{\lambda\left(n_{0}-1\right)}\right)
$$

qui est représentée sur la Figure 3. On y voit à nouveau l'intérêt de travailler dans l'ordre 1 : c'est ce que nous supposerons dans la suite.

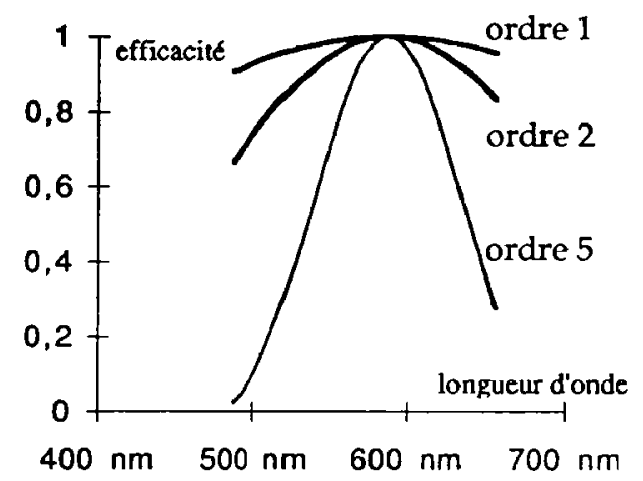

Figure 3. Efficacité en fonction de la longueur d’onde pour un réseau zoné "blazé" dans l'ordre q= 1, 2, 5.

\subsubsection{Réalisation par gravure d l'outil diamanté}

Le tour d'opticien à outil diamanté a maintenant fait son entrée, bien qu'en haut de gamme, dans l'atelier d'optique. L'outil, à tête fine, usine la surface du composant d'optique comme un outil de tour de mécanicien usine une surface métallique, mais produit directement une qualité de surface optiquement polie. La trajectoire en spirale de l'outil se remarque toutefois par la présence de discrets sillons diffractants. Cette technique d'usinage est bien adaptée pour les surfaces asphériques, mais convient également pour la réalisation des profils discontinus de l'optique diffractive.

\subsubsection{Réalisation par masquage}

La technique du masquage est semblable à celle utilisée en microélectronique pour réaliser les circuits logiques, d'où le nom quelque peu trompeur d'optique "binaire" donné aux composants qui en résultent et qui précisément ne peuvent pas se limiter à deux niveaux. La technique, due à Huignard et d'Auria [7], réalise une approximation en escaliers du profil souhaité (voir Figure 2) et comporte les étapes suivantes :

- réalisation par une méthode d'arts graphiques d'un premier masque $\mathbf{M}_{1}$, par exemple sur plaque de verre chromée, et développement chimique pour enlever le chrome aux endroits exposés. La résolution nécessaire étant peu élevée, il n'est en général pas utile de recourir au masquage par faisceau d'électrons. On obtient donc un motif binaire, qui est transparent dans les parties où la profondeur de gravure souhaitable dépasse $h / 2$ selon l'équation (19) et opaque ailleurs (ou réciproquement). 
- Dépôt sur le substrat du composant diffractif d'une couche de résine d'épaisseur $h / 2$, exposition de la résine par le masque $\mathrm{M}_{1}$, développement de la résine : les parties où la gravure doit dépasser $h / 2$ sont maintenant gravées à une profondeur $h / 2$.

- Réalisation d'un masque $\mathrm{M}_{2}$ transparent dans les parties où la profondeur de gravure dépasse $\mathrm{h} / 4$ modulo $\mathrm{h} / 2$.

- Dépôt sur le substrat d'une couche de résine d'épaisseur $h / 4$, exposition et développement, on obtient un composant à 4 niveaux.

- et ainsi de suite jusqu'au dernier masque $M_{M}$, transparent dans les parties où la gravure dépasse $h / 2^{M}$ modulo $h / 2^{M-1}$, et qui foumit après développernent de la résine un composant à $2^{\mathrm{M}}$ niveaux.

Le profil ainsi obtenu est en général transféré de la résine dans le substrat par usinage ionique. Comme les vitesses de gravure de la résine et du substrat par le faisceau d'ions sont en général proportionnelles et non pas égales, il convient en fait de précompenser cet effet en multipliant les hauteurs de résines indiquées ci-dessus par le rapport convenable. Le masquage a l'avantage de fournir des composants de bonne qualité de surface entre les niveaux. Une difficulté liée au masquage est la nécessité d'un alignement particulièrement précis des différentes images de masques sur le composant.

L'efficacité de diffraction de l'ordre 1 résulte de la transformation de Fourier d'une fonction en escalier de la variable $p^{2}$ et a pour expression à la longueur d'onde nominale $\lambda_{0}$ :

$$
\eta_{M}=\operatorname{sinc}^{2}\left(2^{-M}\right)
$$

(On notera que dans cette équation, l'indice de $\eta$ désigne le nombre de masques et non plus l'ordre de diffraction). Le tableau 2 met en évidence l'importance du nombre $M$ de niveaux de masquage. Notamment, un composant vraiment binaire, incapable de distinguer l'ordre 1 de l'ordre -1 , est à proscrire, mais dès $\mathbf{M}=2$ l'ordre utile est nettement privilégié.

Tableau 2. Efficacité maximale d'un composant à M niveaux de masquage.

\begin{tabular}{|c|c|}
\hline M & efficacité \\
\hline 1 & $\overline{0}, 405$ \\
\hline 2 & 0,811 \\
\hline 3 & 0,950 \\
\hline 4 & 0,987 \\
\hline
\end{tabular}

A une longueur d'onde quelconque, l'expression suivante généralise (pour l'ordre de diffraction 1) les équations (20) et (21):

$$
\eta_{M}(\lambda)=\left[N^{-1} \operatorname{sinc} N^{-1} \frac{\sin \pi \varepsilon}{\sin \pi N^{-1} \varepsilon}\right]^{2}, \text { avec } N=2^{M} \text { et } \varepsilon=\frac{\lambda_{o}(n-1)}{\lambda\left(n_{o}-1\right)}-1
$$

Indiquons enfin l'efficacité de diffraction dans l'ordre $\mathrm{Q}$, à la longueur d'onde $\lambda$ d'un réseau à $\mathrm{M}$ niveaux de masques calculé pour fonctionner dans l'ordre $q$ à la longueur d'onde $\lambda_{\mathrm{a}}$ :

$$
\eta_{M, Q}(\lambda)=\left[N^{-1} \operatorname{sinc} Q N^{-1} \frac{\sin \pi \varepsilon}{\sin \pi N^{-1} \varepsilon}\right]^{2}, \text { avec } N=2^{M} \text { et } \varepsilon=q \frac{\lambda_{o}(n-1)}{\lambda\left(n_{o}-1\right)}-Q
$$




\section{CORRECTION D'UNE ABERRATION QUELCONQUE}

L'achromatisation et l'athermalisation ont longtemps été considérées comme les applications essentielles de l'optique hybride. Dans l'un et l'autre cas, la partie diffractive du système se ramène à un réseau zoné, c'est-à-dire à une lentille diffractive, dont le profil de phase est une fonction périodique de $\rho^{2}$. Il n'est cependant pas exclu qu'apparaissent des applications intéressantes ne présentant pas cette périodicité. C'est le cas si l'on sort de l'approximation de Fresnel de l'onde sphérique rappelée au paragraphe 1.2 , c'est aussi le cas si on souhaite corriger une aberration autre que le chromatisme par l'optique diffractive [8].

II convient donc de terminer par une analyse des composants diffractifs pour la synthèse d'une phase non nécessairement périodique qui inclut la présentation des paragraphes précédents comme cas particulier.

Si la conception d'un système optique amène à la conclusion qu'à une longueur d'onde donnée $\lambda_{o}$, un composant diffractif produisant la phase $\phi(x, y)$ est utile, l'expression (4) montre immédiatement que la gravure d'une épaisseur

$$
e(x, y)=\frac{\lambda_{0}}{2 \pi\left(n_{o}-1\right)} \phi(x, y)
$$

fournit la solution. On omettra dans la suite les variables $(x, y)$ pour alléger les notations. La phase $\phi$ n'étant définie que modulo $2 \pi$, toute détermination de e modulo $\frac{q \lambda_{o}}{n_{o}-1}$ est acceptable à cette longueur d'onde, $q$ étant un entier quelconque appelé ordre de diffraction et en général choisi égal à 1 , comme on l'a vu. L'efficacité de diffraction et le chromatisme du composant s'obtiennent alors aisément en exprimant la phase $\phi^{\prime}$ produite en fonction de la phase $\phi$ souhaitée, à une longueur d'onde quelconque et en tenant compte du profil de phase réellement fourni par le procédé de gravure. A titre d'exemple, la Figure 4 présente la phase $\phi$ ' en fonction de $\phi$ à une longueur d'onde $\lambda=0,8 \lambda_{\mathrm{o}}$ dans le cas d'un masquage à trois niveaux. C'est alors une série de Fourier dont la variable est $\phi$ et non pas une variable d'espace qui foumit l'efficacité de diffraction.

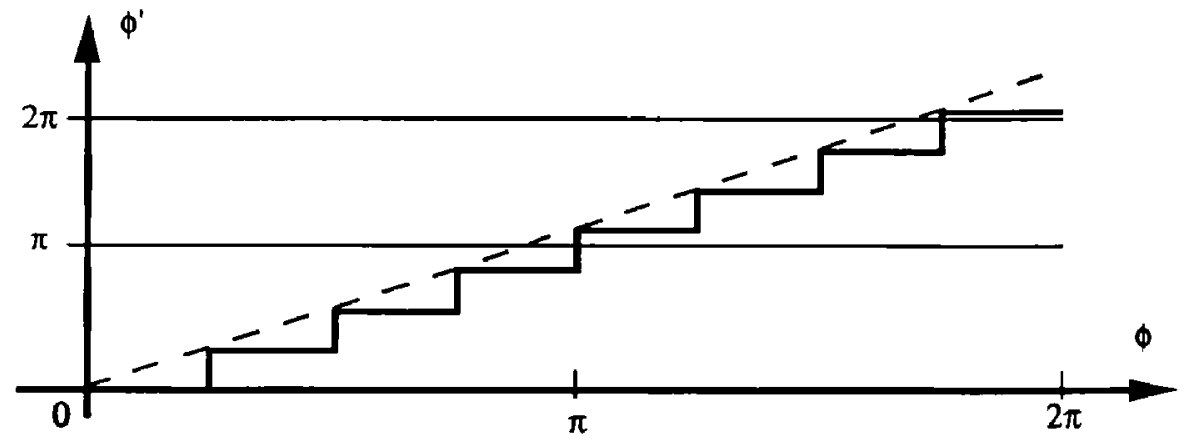

Figure 4. Un exemple de phase réalisée en fonction de la phase de consigne. En pointillés, la droite correspondant à une gravure continue (limite $M$ infini). 


\section{CONCLUSION}

Nous avons examiné les bases de l'approche usuelle de l'optique diffractive dans les systèmes optiques hybrides réfractifs et diffractifs. Leur intérêt pour l'achromatisation des objectifs dans le domaine infrarouge thermique leur a valu d'accéder au marché de l'optique professionnelle ces demières années, et une évolution vers les longueurs d'onde de l'opto-électronique et des télécommunications, puis du visible, est probable. Il reste cependant à résoudre le problème de l'aide à la conception d'optiques hybrides. Notamment, nous avons porté notre antention sur l'ordre utile, en général l'ordre 1, et il convient de veiller à la perturbation apportée par les autres ordres. En effet, du moins dans les composants de phase qui sont de loin les plus nombreux, la lumière qui n'est pas dirigée dans l'ordre utile se perd dans les autres ordres qui occasionnent des images parasites. Actuellement, les logiciels de conception assistée par ordinateur disponibles sur le marché considèrent au mieux cette lumière comme diffusée sous forme d'un fond parasite uniforme, si toutefois ils ne la négligent pas totalement dans le calcul des fonctions de transfert. Il serait possible et utile d'en tenir compte dans l'analyse du système. Au moins pour les composants à faible nombre de zones (voir tableau 1), on pourrait même penser à remplacer l'analyse par sommation de Fourier des ordres de diffraction par une analyse par superposition cohérente des images formées par les différentes zones : le calcul pourrait en être simplifié et donner accès à la variation des rayons de zones et hauteurs de discontinuités comme variables d'optimisation supplémentaires.

\section{Références}

[1] Floquet G., Ann. Ecole Norm., 12 (1883) 47.

[2] voir par exemple "Electromagnetic Theory of Gratings," sous la direction de Petil R., Springer Verlag, Berlin 1980, Topics in Current Physics, volume 22.

[3] Soret J.L., "Sur les phénomènes de diffraction produits par les réseaux circulaires," Archives des Sciences Physiques et Naturelles, 52 (1875) 320-327.

[4] Fresnel A., Ann. Phys. Chim., série 2, volume 1 (1816) 239.

[5] pour plus d'information, voir par exemple Geyl R., "Le calcul des combinaisons", dans "Systèmes optiques", Ecole de la SFO 1991, Roblin G., éditeur scientifique (Editions de Physique, les Ulis, 1992) pp 145-186.

[6] Swanson G.J., "Binary Optics Technology: the Theory and Design of Multi-Level Diffractive Optical Elements", MIT Report, 1989.

[7] d'Auria L., Huignard J.P., Roy A.M. et Spitz E., "Photolithographic Fabrication of Thin Film Lenses," Opt. Commun., 5 (1972) 232-235. Voir aussi Miyamoto K., "The Phase Fresnel Lens," J. Opt. Soc. Am., 51 (1961) 17-20.

[8] La société Melles Griot a très récemment annoncé un triplet hybride achromatique, composé d'un doublet réfractif et d'une gravure diffractive, dont la qualité ne peut être atteinte sans correction d'aberration de sphéricité d'ordre supérieur. La documentation commerciale ne précise toutefois pas le rôle exaci dévolu à la partie diffractive. 